\title{
Application of nasal continuous positive airway pressure to early extubation in very low birthweight infants
}

\author{
B-Horng So, M Tamura, J Mishina, T Watanabe, S Kamoshita
}

\begin{abstract}
Using a preset protocol for early extubation, 50 babies were randomly selected to post-extubation headbox or post-extubation nasal continuous positive airway pressure (N-CPAP). All infants weighed less than $1500 \mathrm{~g}$, had a gestational age of less than 34 weeks, and had been weaning from mechanical ventilation within seven days of life. The criteria for extubation included stable condition, fraction of inspired oxygen $\left(\mathrm{FIO}_{2}\right)$ of $\leqslant 35 \%$, peak inspiratory pressure (PIP) of $\leqslant 15 \mathrm{~cm} \mathrm{H}_{2} \mathrm{O}(1.47 \mathrm{kPa})$, and ventilator rate of $6 /$ minute. Before extubation, a loading dose of aminophylline was given followed by maintenance treatment. If reintubation was not required within 72 hours of the initial extubation the procedure was considered successful. The reintubation criteria included $\mathrm{FIO}_{2}$ of $\geqslant 70 \%$ to maintain arterial oxygen tension $\left(\mathrm{PaO}_{2}\right)$ of $\geqslant 50 \mathrm{~mm} \mathrm{Hg} \mathrm{(6.67}$ $\mathrm{kPa}$ ) or pulse oximetry between $90-96 \%$ and $\mathrm{pH}$ of $<7.25$, and arterial carbon dioxide tension $\left(\mathrm{PACO}_{2}\right)$ of $>60 \mathrm{~mm} \mathrm{Hg}$ $(8.00 \mathrm{kPa})$ and severe or recurring apnoea. The overall success rate of early extubation was $66 \%(33 / 50)$. The individual successful extubation rate of post-extubation in the N-CPAP group and the postextubation headbox group were $84 \%$ $(21 / 25)$ and $48 \%(12 / 25)$, respectively $\left(\mathbf{p}=0.017 ; \chi^{2}\right)$. There were no significant differences in clinical characteristics between the two groups. The most common cause of failure in early extubation was apnoea, and most occurred in the headbox group (9/12).

These results suggest that application of N-CPAP to a preset protocol for extubation can achieve a better success rate of early extubation in very low birthweight (VLBW) infants.

(Arch Dis Child 1995; 72: F191-F193)
\end{abstract}

Keywords: N-CPAP, very low birthweight, early extubation.

Reintubation of the very low birthweight (VLBW) infant after recovery from respiratory distress is common. Therefore, there is a tendency to prolong mechanical ventilation, with a low ventilator rate, in the hope that the infant's condition will stabilise before extubation. Under these circumstances some infants will develop complications, including bronchopulmonary dysplasia (BPD), ${ }^{1}$ subglottic stenosis, ${ }^{23}$ infection, increasing the risk of morbidity and mortality. ${ }^{4}$ Several reports have described the criteria for extubation, based on pulmonary function test. ${ }^{5-8}$ Pulmonary function testing, however, is not always routinely available, so we have designed a protocol for early extubation of VLBW infants. Two methods are usually used in post-extubation management - the headbox technique and nasal continuous positive airway pressure $(\mathrm{N}$ CPAP). Because we have experience of using $\mathrm{N}-\mathrm{CPAP}$ as an alternative treatment to the ventilator in respiratory distress syndrome (RDS), ${ }^{910}$ we tried in this study to estimate whether the concomitant use of the protocol and post-extubation N-CPAP would be more effective for early extubation in VLBW infants than the post-extubation headbox.

\section{Methods}

Infants with the following characteristics were enrolled into this study after informed consent had been obtained from their parents: (i) birthweight of $<1500 \mathrm{~g}$; (ii) gestational age of $<34$ weeks; (iii) mechanical ventilation after intubation initiated during the first few hours of life; (iv) weaning started within seven days of life; (v) absence of overwhelming infection and congenital abnormalities. All infants followed the protocol for early extubation as soon as their condition had stabilised. The $\mathrm{FIO}_{2}$ was lowered by 2 to $10 \%$ to at least less than $35 \%$ before extubation to maintain a $\mathrm{PaO}_{2}$ between 50 and $70 \mathrm{~mm} \mathrm{Hg}(6.67$ and $9.33 \mathrm{kPa})$. The peak inspiratory pressure (PIP) was reduced by 2 to $5 \mathrm{~cm} \mathrm{H}_{2} \mathrm{O}$ to $15 \mathrm{~cm} \mathrm{H}_{2} \mathrm{O}$, and subsequently PIP was held constant unless breathing became disturbed, when it was lowered by $2 \mathrm{~cm} \mathrm{H}_{2} \mathrm{O}$. The positive end expiratory pressure (PEEP) was maintained at $5 \mathrm{~cm} \mathrm{H}_{2} \mathrm{O}$ during the period of mechanical ventilation. The intermittent mandatory ventilation (IMV) rate was decreased by 5 to 10 per minute for maintaining the $\mathrm{PACO}_{2}$ at $<50 \mathrm{~mm} \mathrm{Hg}(6.67 \mathrm{kPa})$, until an IMV rate of $30 / \mathrm{minute}$ was reached, and subsequently by 2 to 5 per minute until reaching a rate of $10 /$ minute. Then aminophylline was started with a loading dose of $4 \mathrm{mg} / \mathrm{kg}$ intravenously or by mouth followed by maintenance dose of 1 to $2 \mathrm{mg} / \mathrm{kg}$ every 12 hours adapted to maintain the serum concentration between 8 and $15 \mu \mathrm{g} / \mathrm{ml}$. Extubation was undertaken when the IMV rate reduced to $6 / \mathrm{min} 12$ to 24 hours after the loading dose of aminophylline. 
Table 1 Clinical characteristics

\begin{tabular}{lcc}
\hline & $N-C P A P$ & Headbox \\
\hline Gestational age (weeks) & $28 \cdot 1(1 \cdot 9)(25-33)$ & $28 \cdot 4(2 \cdot 1)(24 \cdot 3-33)$ \\
Birthweight (g) & $1090(231)(610-1490)$ & $1130(231)(690-1490)$ \\
Apgar score at 1 minute & $3 \cdot 6(2 \cdot 2)(0-7)$ & $4 \cdot 3(1 \cdot 5)(1-8)$ \\
at 5 minutes & $7 \cdot 0(1 \cdot 4)(4-9)$ & $7 \cdot 2(2 \cdot 0)(1-9)$ \\
Extubation age (days) & $5 \cdot 1(2 \cdot 6)(0 \cdot 3-9)$ & $6 \cdot 0(2 \cdot 2)(1-9)$ \\
\hline
\end{tabular}

Values are mean (SD) (range).

Before extubation the infants were given CPAP $5 \mathrm{~cm} \mathrm{H}_{2} \mathrm{O}$ through the endotracheal tube for one hour. If respiratory acidosis $(\mathrm{pH}$ $\left.<7 \cdot 25, \mathrm{PACO}_{2}>60 \mathrm{~mm} \mathrm{Hg}\right)(8.00 \mathrm{kPa})$ and severe or frequent apnoea did not develop, the infant was extubated. After extubation, it is not unusual to register a fall in $\mathrm{PACO}_{2}$ below that noted with endotracheal tube CPAP. This is probably attributable to the lower airway resistance after extubation. When extubation and spontaneous breathing were well established, aminophylline was stopped after 35 weeks of gestational age.

Before extubation, each infant was randomly assigned to either N-CPAP or headbox. Infants assigned to headbox received an appropriate concentration of humidified oxygen or air for at least 24 hours after the extubation. Infants assigned to N-CPAP used the CPAP system without the ventilator as reported before. ${ }^{10}$ In brief, the system consists of (i) an oxygen blender with a flow meter, (ii) a heated humidifier, (iii) an inspiratory tubing with a thermometer inserted in the midway, (iv) a nasal prong ${ }^{9}$ with a manometer connected to the expiratory end, (vi) an expiratory tubing, and (vii) a bottle containing sterile distilled water to a depth of $7 \mathrm{~cm}$. We achieved the desired level of CPAP ( $5 \mathrm{~cm} \mathrm{H}_{2} \mathrm{O}$ ) by simply immersing the expiratory tubing in water to a desired depth $(5 \mathrm{~cm})$.

After extubation transcutaneous $\mathrm{PO}_{2}$ and $\mathrm{PCO}_{2}$, pulse oxymetry saturation, heart and respiratory rate were monitored continuously. Arterial blood gases were checked when clinically indicated. All infants received chest physiotherapy to prevent atelectasis. ${ }^{11} 12$ Extubation was considered successful if the reintubation was not required within 72 hours of the initial extubation. The criteria for reintubation were (i) $\mathrm{PaO}_{2}<50 \mathrm{~mm} \mathrm{Hg}$ $(6.67 \mathrm{kPa})$ with $\mathrm{FIO}_{2} \geqslant 70 \%$; (ii) a respiratory acidosis with $\mathrm{pH}<7 \cdot 25$ and $\mathrm{PACO}_{2}$ $>60 \mathrm{~mm} \mathrm{Hg}(8.0 \mathrm{kPa})$; (iii) severe or frequent

Table 2 Comparison of success (S) and failure $(F)$ in both groups

\begin{tabular}{|c|c|c|c|c|}
\hline & \multicolumn{2}{|l|}{$N-C P A P$} & \multicolumn{2}{|l|}{ Headbox } \\
\hline & $S(n=21)$ & $F(n=4)$ & $S(n=12)$ & $F(n=13)$ \\
\hline $\begin{array}{l}\text { Gestational age (weeks) } \\
\text { Birth weight (g) } \\
\text { Extubation age (days) } \\
\text { Surfactant supplement } \\
\text { Success rate } \\
\text { Sex (M/F) } \\
\text { PDA } \\
\text { IVH } \\
\text { BPD } \\
\text { NEC } \\
\text { ROP }\end{array}$ & $\begin{array}{c}28 \cdot 3 \pm 2 \cdot 0 \\
1100 \pm 225 \\
4 \cdot 7 \pm 2 \cdot 8 \\
10(48) \\
21(84) \\
11 / 10 \\
7(33) \\
1(5) \\
1(5) \\
1(5) \\
1(5)\end{array}$ & $\begin{array}{c}27 \cdot 3 \pm 1 \cdot 0 \\
1040 \pm 290 \\
7 \cdot 0 \pm 1 \cdot 6 \\
4(100) \\
- \\
2 / 2 \\
2(50) \\
1(25) \\
2(50) \\
0 \\
1(25)\end{array}$ & $\begin{array}{c}28 \cdot 8 \pm 2 \cdot 1 \\
1143 \pm 199 \\
5 \cdot 7 \pm 2 \cdot 1 \\
8(67) \\
12(48) \\
5 / 7 \\
5(42) \\
1(8) \\
2(17) \\
1(8) \\
1(8)\end{array}$ & $\begin{array}{c}28 \cdot 0 \pm 1 \cdot 9 \\
1124 \pm 255 \\
6 \cdot 2 \pm 2 \cdot 2 \\
7(54) \\
- \\
9 / 4 \\
5(38) \\
2(15) \\
3(23) \\
0 \\
1(8)\end{array}$ \\
\hline
\end{tabular}

Values are mean $\pm S D$ or number (per cent). There were no significant differences within each group (N-CPAP or headbox). PDA, patent ductus arteriosus. IVH, intraventricular haemorrhage. BPD, bronchopulmonary dysplasia. NEC, necrotising enterocolitis. ROP, retinopathy of prematurity. apnoea. Apnoea was defined as a respiratory pause of $\geqslant 20$ seconds or less, but associated with bradycardia or cyanosis. Severe apnoea was considered if three or more apnoeic episodes in one hour or one requiring vigorous stimulation or mask and bag ventilation developed.

According to previous experience, about $40 \%$ of infants similar to those enrolled into this study are successfully extubated to a headbox. We anticipated that using N-CPAP could double the success rate to about $80 \%$. If so, then post-extubation N-CPAP could be adopted as a routine practice in our unit.

The statistical differences were assessed using the unpaired, two tailed Student's $t$ test or the $\chi^{2}$ test as appropriate. A p value of less than 0.05 was considered significant.

\section{Results}

Fifty infants were included in the study, 25 assigned to post-extubation headbox and 25 to $\mathrm{N}$-CPAP. The infants of both groups were comparable in terms of gestational age, birthweight, Apgar scores, postnatal age at extubation, incidence of patent ductus arteriosus (PDA), incidence of intraventricular haemorrhage (IVH), incidence of necrotising enterocolitis (NEC), surfactant supplement, use of aminophylline and gender (tables 1 and 2).

The extubation was successful in $21(84 \%)$ infants in the N-CPAP group and in $12(48 \%)$ infants in the headbox group $\left(p=0.017, \chi^{2}\right)$. The clinical characteristics of gestational age, birthweight, postnatal age at extubation, PDA, IVH, diagnosis of BPD (defined as oxygen dependency at 28 days with characteristic changes on a chest radiograph) and gender of the successfully extubated infants and of those who were not showed no significant difference (table 2).

The causes for and the number of infants not successfully extubated in each group are shown in table 3 . There were no significant differences between the two groups. The most common cause of failure in early extubation was apnoea, and most occurred in the headbox group (9/12).

\section{Discussion}

The overall success rate in this study using the protocol for early extubation was $66 \%(33 / 50)$. We did not measure pulmonary function before extubation, therefore we are unable to comment on whether this testing would improve our success rate. In a study of extubating VLBW infants based on pulmonary function test, $79.5 \%(58 / 73)$ infants were successfully extubated. ${ }^{13}$ We achieved a similar success rate based on a preset protocol for early extubation in VLBW infants. Our protocol is based on the precise criteria for early weaning from mechanical ventilation and use of aminophylline. Aminophylline has proved effective in the prevention of post-extubation respiratory failure. ${ }^{14-16}$ Pulmonary function tests have a definite role in understanding the pulmonary mechanics of various respiratory diseases, but the practical values of such measurements 
Table 3 Causes and numbers of extubation failure

\begin{tabular}{lll}
\hline & $N-C P A P(n=4)$ & Headbox $(n=13)$ \\
\hline FiO $_{2}>0 \cdot 7$ required & 1 & 1 \\
pH $<7 \cdot 25$, PACO $_{2}>60$ & 0 & 1 \\
Apnoea & 3 & 9 \\
Atelectasis & 0 & 2 \\
\hline
\end{tabular}

before extubation in VLBW infants has been questioned. 56

The individual success rate of infants in the post-extubation N-CPAP group and those in the post-extubation headbox group are $84 \%$ and $48 \%$, respectively. Post-extubation N-CPAP was more successful in preventing extubation failure. Several studies of the application of N-CPAP to extubation have been reported. Some reported a higher success rate, ${ }^{17-19}$ while another did not. ${ }^{20}$ The conflicting results might be attributable firstly to the gestational ages of the infants. But several studies applying N-CPAP post-extubation on much more premature infants also had a higher successful extubation rate. ${ }^{18} 1921$ The second factor could be the level of nasal CPAP used. The best CPAP level may be different for each infant and the optimal CPAP ${ }^{22}{ }^{23}$ requires serial determination of cardiovascular and respiratory response in each infant. We usually set the $\mathrm{CPAP}$ at $5 \mathrm{~cm} \mathrm{H}_{2} \mathrm{O}$. Severe inspiratory retractions may point to a decreasing compliance as a result of the falling functional reserve capacity (FRC) and an indication to increase CPAP. For these infants, CPAP is increased to $7 \mathrm{~cm} \mathrm{H}_{2} \mathrm{O}$ and the response is assessed by observing retractions and oxygenation. We don't use CPAP over $7 \mathrm{~cm} \mathrm{H}_{2} \mathrm{O}$. CPAP should be decreased if a chest radiograph reveals overexpansion of lungs, particularly in the infants with $\mathrm{CO}_{2}$ retention.

The extubation failure rate differed significantly between infants who were assigned to N-CPAP or headbox. The most common cause of failure in both groups was apnoea, and most of this occurred in the headbox group. Many premature infants do not require oxygen as much as they require CPAP for their respiratory distress. This can be shown clinically by the fact that the decreased oxygen saturation on a pulse oxymeter and the lowered $\mathrm{PO}_{2}$ on a transcutaneous monitor rise immediately after closing the infant's mouth to decrease the escape of CPAP but without increasing $\mathrm{FIO}_{2}$ or CPAP. If a premature infant is extubated to headbox, the alveoli are prone to collapse during expiration, breathing becomes increasingly difficult, and the available surfactant in the alveoli is rapidly depleted. ${ }^{24}$ When an infant is placed on N-CPAP immediately after extubation, this cycle can be interrupted and post-extubation respiratory failure can be prevented. N-CPAP works by improving FRC, preventing atelectasis, and reducing the breathing work. A $5 \mathrm{~cm} \mathrm{H} \mathrm{H}_{2} \mathrm{O}$-CPAP produced a decrease in supraglottic resistance and total pulmonary resistance considered to be a primary means of reducing apnoea in premature infants. ${ }^{23}$
In conclusion, this study shows that a preset protocol can achieve early extubation in VLBW infants. And this effect can be further reinforced by applying N-CPAP immediately after extubation to prevent post-extubation respiratory failure. Moreover, we used the N-CPAP system without a ventilator, therefore addressing the shortage of ventilators in neonatal intensive care.

Part of this article had been presented at The 8th Congress of The Federation of The Asia/Oceania Perinatal Societies, Taipei, Taiwan. September 1994.

1 Overstreet DW, Jackson JC, Belle van G, Truog WE. Estimation of mortality risk in chronically ventilated infants with bronchopulmonary dysplasia. Pediatrics 1991; 88: 1153-60.

2 Fan LL, Flynn JW, Pathak DR. Risk factors predicting laryngeal injury in intubated neonates. Crit Care Med 1983; 11: 431-3.

3 Abert DM, Mills RP, Fysh J, Gamsu H, Thomas JN. Endoscopic examination of the neonatal larynx at extubation: a prospective study of variables associated with laryngeal damage. Int $\mathcal{F}$ Pediatr Otorhinolaryngol 1990; 20:

4 Moriette G, Gaudebout C, Clement A, Boule M, Bion B, Relier JP, et al. Pulmonary function at one year of age in survivors of neonatal respiratory distress: A multivariate analysis of factors associated with sequelae. Pediatr Pulmonol 1987; 3: 242-50.

5 Balsan MJ, Jones JG, Watchko JF, Guthrie RF. Measurement of pulmonary mechanics prior to the elective extubation of neonates. Pediatr Pulmonol 1990; 9: 238-43.

6 Veness-Meehan KA, Richter S, Davis JM. Pulmonary function testing prior to extubation in infants with respiratory tion testing prior to extubation in infants with respirt

7 Shimada Y, Yoshida I, Tanaka K, Yamazaki T, Kumon K. Crying vital capacity and maximal inspiratory pressure as clinical indicators of readiness for weaning of infants less than a year of age. Anesthesiol 1979; 51: 456-9.

8 Osada A. Peak crying inspiratory flow rate (PCIFR) as a criterion for extubation. Fournal of the fapanese Paediatric Society 1991; 95: 2015-22

9 So B-H, Sibuya K, Tamura M, Watanabe H, Kamoshita S. Clinical experience in using a new type of nasal prong for administration of N-CPAP. Acta Paediatr Fpn 1992; 34: 328-33.

10 So B-H, Tamura M, Kamoshita S. Nasal Continuous positive airway pressure following surfactant replacement for the treatment of neonatal respiratory distress syndrome. the treatment of neonatal respiratory

11 Finer NN, Moriartey RR, Boyd J, Phillips HJ, Steward AR, Ulan O. Postextubation atelectasis: retrospective review and a prospective controlled study. $\mathcal{F}$ Pediatr 1974; 94: $110-3$.

12 Odita JC, Kayyali M, Ammari A. Post-extubation atelectasis in ventilated newborn infants. Pediatr Radiol 1993; 23: 183-5.

13 Hasegawa H. Pulmonary function test in infants. fournal of the Fapan Society for Premature and Newborn Medicine 1993; 5: 41-58.

14 Kuzemko JA, Paala J. Apneic attacks in the newborn treated with aminophylline. Arch Dis Child 1973; 48: 404-6.

15 Viscardi RM, Faix RG, Nicks J, Grasela TH. Efficacy of theophylline for prevention of post-extubation respiratory failure in very low birth weight infants. Pediatrics 1985; 107: 469-72.

16 Durand DJ, Goodman A, Ray P, Ballard RA, Clyman RI. The theophylline treatment in the extubation of infants weighing less than 1250 grams: a controlled trial. Pediatrics 1987; 80: 684-8.

17 Engelke SC, Roloff DW, Kuhns LR. Post extubation nasal continuous positive airway pressure: a prospective controlled study. Am $\mathcal{F}$ Dis Child 1982; 136: 359-61.

18 Andrèasson $B$, Lindroth $M$, Svenningsen NW, Jonson $B$. Effects on respiration of CPAP immediately after extubation in the very preterm infant. Pediatr Pulmonol 1988; 4: 213-8.

19 Higgins RD, Richter SE, Davis JM. Nasal continuous positive airway pressure facilitates extubation of very low birth weight neonates. Pediatrics 1991; 88: 999-1003.

20 Chan V, Greenough A. Randomized trial of methods of extubation in acute and chronic respiratory distress. Arch Dis Child 1993; 68: 570-2.

21 Wung JT, Koons AH, Driscoll JM Jr, James LS. Changing incidence of bronchopulmonary dysplasia. F Pediatr 1979; 95: 845-7.

22 Suter PM, Fairly HB, Isewnberg MD. Optimum endexpiratory airway pressure in patients with acute pulmonary failure. N Engl $\mathcal{F}$ Med 1975; 292: 284-9.

23 Miller MJ, DiFiore JM, Strohl KP, Martin RJ. Effects of nasal CPAP on supraglottic and total pulmonary resistance in preterm infants. F Appl Physiol 1990; 68: 141-6.

24 Brady JP, Gregory GA. Assisted ventilation. In: Klaus MH, Fanaroff AA, eds. Care of the high-risk neonate. Philadelphia: WB Saunders, 1993: 239-40. 